\section{Hitting histone demethylases}

\section{By Joanne Kotz, Senior Editor}

A team from GlaxoSmithKline plc and the Structural Genomics Consortium has identified the first selective and potent histone demethylase inhibitor and shown it dampens the macrophage inflammatory response. ${ }^{1}$ The pharma now is exploring the therapeutic applications of the inhibitor in autoimmune and inflammatory diseases.

Histone methyltransferases add methyl groups to histone lysine residues, which can then be removed by histone demethylases. These two enzyme classes work in concert to regulate gene transcription.

Academics and companies have identified histone methyltransferase inhibitors, suggesting the enzyme class is generally druggable. However, whether potent and selective inhibitors could be developed against histone demethylases has remained an open question.

Previous efforts, including work at SGC, identified histone demethylase inhibitors that had either in vitro selectivity or potencybut not both-and none had sufficient cellular activity, said Udo Oppermann, a group leader in epigenetics and inflammation at the SGC and professor in molecular biology and deputy director of the Institute of Musculoskeletal Sciences at the University of Oxford. Oppermann was a coauthor on the study.

The SGC leads a public-private partnership, which includes GSK, five other pharmas and additional academic groups, that is developing open-access chemical probes that modulate epigenetic proteins.

The GSK-SGC team set out to identify a potent and selective inhibitor of the histone demethylase jumonji domain containing 3 (JMJD3; KDM6B), which removes methyl groups specifically from trimethylated lysine 27 on histone 3 (H3K27me3).

Proinflammatory stimuli such as lipopolysaccharide (LPS) induce expression of JMJD3 in macrophages. The histone demethylase then participates directly in activating the transcription of more than half of the LPS-induced genes. ${ }^{2,3}$

First, the team obtained a weakly active hit against JMJD3 via a high throughput screen of GSK's library of two million compounds. The researchers then used structure-based design to optimize the hit to the lead molecule, GSK-J1, which inhibited JMJD3 with an $\mathrm{IC}_{50}$ of $60 \mathrm{nM}$.

In addition to its potency, the molecule also had good selectivity-it showed little to no activity against a panel of about 10 other jumonji histone demethylases.

GSK-J1 did show activity against a very closely related histone demethylase called lysine-specific demethylase 6A (KDM6A; UTX). Like JMJD3, UTX catalyzes the demethylation of H3K27me3.
In LPS-stimulated primary human macrophages from healthy volunteers, a prodrug of GSK-J1 with improved cell permeability blocked the expression of half of the LPS-induced cytokines, including tumor necrosis factor- $\alpha$ (TNF- $\alpha$ ). Subsequent experiments showed that inhibiting both JMJD3 and UTX was required for GSK-J1's activity.

Finally, the team asked whether GSK-J1 had activity in a disease context. In macrophages obtained from patients with rheumatoid arthritis (RA), the prodrug blocked TNF- $\alpha$ production.

Data were published in Nature.

"GSK-J1 is the first truly selective jumonji inhibitor and hence represents an important step in demonstrating the tractability of the jumonji family for drug discovery. It is also the first selective H3K27 demethylase inhibitor reported and as such provides a chemical tool to further explore the biology and therapeutic potential of H3K27 demethylases," said Laurens Kruidenier, first author on the paper and lead biologist in GSK's immuno-inflammation EpiNova DPU.

"This is the first paper that shows it is possible to make inhibitors with high affinity and specificity" against jumonji histone demethylases, agreed Kristian Helin, director of the Biotech Research \& Innovation Centre at the University of Copenhagen and CSO of EpiTherapeutics ApS.

EpiTherapeutics is targeting histone methyltransferases and histone demethylases in cancer. Helin told SciBX that EpiTherapeutics has undisclosed
"GSK-J1 is the first truly selective jumonji inhibitor and hence represents an important step in demonstrating the tractability of the jumonji family for drug discovery." - Laurens Kruidenier, GlaxoSmithKline plc inhibitors of other jumonji histone demethylases with potency and selectivity profiles that are similar to those of GSK-J1.

"Dual pharmacological inhibition of JMJD3 and UTX results in the amelioration of a wide array of important proinflammatory cytokines that includes TNF- $\alpha$. The mechanistic evaluation of JMJD3/UTX inhibitors in animal models of inflammation" will be an important next step, said Jose Lora, senior director of preclinical sciences at epigenetics drug discovery company Constellation Pharmaceuticals Inc.

\section{Casting a wide net}

"GSK continues to drive focused validation efforts with GSK-J1 in a variety of in vitro and ex vivo disease models, internally and through its collaborations," wrote Kruidenier and Rab Prinjha, head of the EpiNova DPU, in a joint email to SciBX.

These studies will include determining the differential effects of GSK-J1 versus existing anti-inflammatory treatments including antiTNF- $\alpha$ antibodies.

Kruidenier and Prinjha said GSK also will compare GSK-J1 with the pharma's bromodomain and extra terminal domain (BET) inhibitor, another epigenetic target that regulates inflammation. 


\section{ANALYSIS}

In 2010, GSK reported in Nature the identification of an inhibitor of BET bromodomains and showed that the compound blocked a subset of the macrophage inflammatory response that did not include TNF- $\alpha{ }^{4}$

Histone demethylases and bromodomains are not the only epigenetic families that GSK is exploring. In 2011, the pharma partnered with Epizyme Inc. to target an undisclosed set of histone methyltransferases. ${ }^{5}$

In addition to these internal and collaborative efforts, Kruidenier and Prinjha said that "GSK-J1 was developed in partnership with the SGC with a view to enabling evaluation by the wider scientific community. GSK-J1 is being made available [through the SGC] to researchers who wish to investigate its role in chromatin biology in their own therapeutic areas."

GSK declined to disclose the patent status of the work.

Kotz, J. SciBX 5(31); doi:10.1038/scibx.2012.805

Published online Aug. 9, 2012

\section{REFERENCES}

1. Kruidenier, L. et al. Nature; published online July 29, 2012; doi:10.1038/nature11262

Contact: David M. Wilson, GlaxoSmithKline plc R\&D, Stevenage, U.K. e-mail: david.m.wilson@gsk.com

2. De Santa, F. et al. Cell 130, 1083-1094 (2007)

3. De Santa, F. et al. EMBO J. 28, 3341-3352 (2009)

4. Nicodeme, E. et al. Nature 468, 1119-1123 (2010)

5. Bouchie, A. \& Fulmer, T. BioCentury 20, A1-A5; Jan. 23, 2012

\section{COMPANIES AND INSTITUTIONS MENTIONED}

Constellation Pharmaceuticals Inc., Cambridge, Mass.

EpiTherapeutics ApS, Copenhagen, Denmark

Epizyme Inc., Cambridge, Mass.

GlaxoSmithKline plc (LSE:GSK; NYSE:GSK), London, U.K.

Structural Genomics Consortium, Oxford, U.K.

University of Copenhagen, Copenhagen, Denmark

University of Oxford, Oxford, U.K. 